\title{
ORIGINAL ARTICLE \\ Relative strength of fine-scale spatial genetic structure in paternally vs biparentally inherited DNA in a dioecious plant depends on both sex proportions and pollen-to-seed dispersal ratio
}

\author{
IJ Chybicki ${ }^{1}, \mathrm{M}$ Dering ${ }^{2}, \mathrm{G}$ Iszkuło $^{2,3}, \mathrm{~K} \mathrm{Meyza}^{1}$ and J Suszka ${ }^{2}$
}

In plants, the spatial genetic structure (SGS) is shaped mainly by gene dispersal and effective population density. Among additional factors, the mode of DNA inheritance and dioecy influence SGS. However, their joint impact on SGS remains unclear, especially in the case of paternally inherited DNA. Using theoretical approximations and computer simulations, here we showed that the relative intensity of SGS measured in paternally and biparentally inherited DNA in a dioecious plant population depends on both the proportion of males and the pollen-to-seed dispersal ratio. As long as males do not prevail in a population, SGS is more intense in paternally than biparentally inherited DNA. When males prevail, the intensity of SGS in paternally vs biparentally inherited DNA depends on the compound effect of sex proportions and the pollen-to-seed dispersal ratio. To empirically validate our predictions, we used the case of Taxus baccata, a dioecious European tree. First, we showed that mitochondrial DNA (mtDNA) in T. baccata is predominantly (98\%) paternally inherited. Subsequently, using nuclear DNA (nuDNA) and mitochondrial microsatellite data, we compared the fine-scale SGS intensity at both marker types in two natural populations. The population with equal sex proportions showed stronger SGS in mtDNA than in nuDNA. On the other hand, we found lower SGS intensity in mtDNA than in nuDNA in the population with $67 \%$ males. Thus, the empirical results provided good support for the theoretical predictions, suggesting that knowledge about SGS in paternally vs biparentally inherited DNA may provide insight into effective sex proportions within dioecious populations.

Heredity (2016) 117, 449-459; doi:10.1038/hdy.2016.65; published online 31 August 2016

\section{INTRODUCTION}

Nonrandom distribution of individual alleles at a local spatial scale, often referred to as the fine-scale spatial genetic structure (SGS), is a common phenomenon within plant populations (Vekemans and Hardy, 2004). In sexually reproducing plants, SGS measured at neutral genomic regions is shaped mainly by two factors: gene dispersal capability and effective population density (Hardy and Vekemans, 1999; Rousset, 2000). Among others, the effective density depends on the effective number of parents (Vekemans and Hardy, 2004) and thus can differ substantially among different plant populations because of differences in distributions of individual reproductive success (Lyons et al., 1989), whereas gene dispersal capability depends on seed and pollen dispersal distances (Crawford, 1984).

Total gene dispersal is highly influenced by the mode of inheritance of a genome (Hamilton and Miller, 2002). In the case of the nuclear genome, as long as sexual reproduction takes place, gametes of both sexes participate in gene transmission. Consequently, nuclear genes are dispersed both through seeds and pollen. However, because ovules and sperm may or may not participate in the transmission of cytoplasmic genomes, seed and pollen dispersal capabilities may or may not influence the total dispersal capability of mitochondrial or chloroplast genes (Ennos, 1994). For example, cytoplasmic genomes are generally maternally inherited in angiosperms (Rebould and Zeyl, 1994). Thus, SGS in chloroplast DNA (cpDNA) and mitochondrial DNA (mtDNA) relies entirely on seed dispersal in this group, whereas pollen dispersal has no impact on the observed SGS. On the other hand, in the Pinaceae family, cpDNA is transmitted paternally whereas mtDNA is transmitted maternally (Mogensen, 1996). Consequently, cpDNA is dispersed both through seeds and pollen and mtDNA only through seeds. As a result, the levels of genetic differentiation among plant populations tend to vary among nuclear, chloroplast and mitochondrial markers (Petit et al., 2005).

A recent comparative study showed that dioecious Ficus species tend to exhibit stronger SGS than monoecious congeners (Nazareno et al., 2013). The authors identified several factors that might be responsible for shaping different potentials for long-distance pollen dispersal in dioecious and monoecious fig species. Interestingly, a broader comparison of SGS among different plant taxa showed that dioecy promotes the development of stronger SGS (Nazareno et al., 2013). Nonetheless, because dioecy tends to increase distances between males and females compared with those of a monoecious species, it would rather weaken the intensity of SGS (Schroeder et al., 2014). Because

${ }^{1}$ Department of Genetics, Kazimierz Wielki University, Bydgoszcz, Poland; ${ }^{2}$ Institute of Dendrology, Polish Academy of Sciences, Kórnik, Poland and ${ }^{3}$ Faculty of Biological Sciences, University of Zielona Góra, Zielona Góra, Poland

Correspondence: Dr IJ Chybicki, Department of Genetics, Institute of Experimental Biology, Kazimierz Wielki University, Chodkiewcza 30 , 85064 Bydgoszcz, Poland.

E-mail: igorchy@ukw.edu.pl

Received 14 December 2015; revised 4 July 2016; accepted 6 July 2016; published online 31 August 2016 
SGS is a compound effect of dispersal and effective density, one can postulate that, in order to counterbalance the effect of increased dispersal, the presence of two sexes decreases the effective population density. The theory of population genetics enables prediction that under equal sex proportions the effective density of a dioecious population would be approximately equal to that of an ideal monoecious population. However, any bias in sex proportions decreases the effective population size, having a negative impact on the effective density (Vandepitte et al., 2010). In reality, many dioecious plants exhibit deviations from the 1:1 sex ratios for a number of reasons. In a recent review, Field et al. (2013) showed that male bias is strongly associated with longevity, biotic seed dispersal and production of fleshly fruits. On the contrary, female bias is associated with abiotic pollen dispersal and the presence of sex chromosomes. In effect, in many dioecious species SGS can be more pronounced compared with monoecious plants. However, although dioecy is generally recognised as an important factor of SGS (Barluenga et al., 2011; Nazareno et al., 2013), to our knowledge the role of sex ratios has not been thoroughly addressed.

When considered separately, the impact of the mode of DNA inheritance and dioecy on the SGS development is predictable to some extent. On the contrary, their joint impact on SGS remains less obvious. In the case of maternally inherited DNA, total gene dispersal is equal to seed dispersal (Ennos, 1994; Petit et al., 2005). Therefore, maternally inherited genes are typically characterised by a shorter dispersal compared with biparentally inherited genes. Moreover, the effective density for maternally inherited genes is on average only half of that for biparentally inherited genes. Both effects lead to the expectation that SGS in maternally inherited DNA should be much stronger than that in biparentally inherited DNA (Petit et al., 2005). However, cytoplasmic DNA shows paternal inheritance in many gymnosperms (Chesnoy, 1987; Mogensen, 1996). In the case of paternally transmitted DNA, genes are dispersed first through pollen and then through seeds. In this respect, they resemble to some degree biparentally inherited nuclear genes, although dispersal capabilities of those two gene types are not equal (Hamilton and Miller, 2002; see also the next section). However, unlike nuclear genes, paternally inherited DNA is transmitted from male parents only and thus should be exposed to higher genetic drift (or higher identity by descent) compared with nuclear genes. As gene dispersal and genetic drift operate in opposite directions in the process of isolation by distance, the effects of dispersal and sex ratios on the relative strength of SGS in paternally and biparentally inherited DNA in a dioecious population are supposed to be rather complex. The question of whether the relative intensity of SGS in paternally and biparentally inherited DNA is predictable under dioecy remains open. Nonetheless, as dioecy is relatively common in gymnosperms compared with angiosperms (54\% vs $6 \%$ of all the species; Givnish, 1980; Renner and Ricklefs, 1995), knowledge about the relative strength of SGS in paternally and biparentally inherited DNA may be potentially helpful in understanding demographic processes and effective sex ratios in gymnosperms.

This study addresses the problem of the relative intensity of SGS in paternally vs biparentally inherited DNA in a dioecious plant population, aiming to resolve whether a balance between dispersal and genetic drift leads to stable and predictable patterns of relative intensity of SGS in paternally and biparentally inherited DNA under dioecy. First, to obtain the approximate prediction of the relative SGS intensity, we reformulated the well-known expectations based on the isolation-by-distance process (Hardy and Vekemans, 1999; Rousset, 2000). Subsequently, the prediction was validated through computer simulations. In addition, using the case study of Taxus baccata, a European dioecious gymnosperm, the study aimed to assess whether the information about relative SGS intensity can be useful for inferring about demographic processes within wild populations.

\section{MATERIALS AND METHODS}

\section{Theoretical approximation}

The aim of this section is to derive the expected relative intensity of SGS in paternally vs biparentally inherited DNA. In plants, SGS is typically assessed as the relationship between genetic similarity and physical distance between two individuals, usually referred to as a correlogram. Thanks to the previous theoretical work (Hardy and Vekemans, 1999; Rousset, 2000), the pure isolation-by-distance process is known to produce a linear-form relationship between the relatedness coefficient and the logarithm of distance between individuals, with the slope parameter being the function of dispersal and demographic parameters. Consequently, the slope of the regression line $(b)$ was proposed to quantify the intensity of SGS (formally as the $S p$ index proportional to $-b$; Vekemans and Hardy, 2004). Following this idea, in order to quantify the relative intensity of SGS in paternally and biparentally inherited genes, we targeted the expected ratios of slopes of regression functions for two gene types in a single dioecious population evolving according to the isolationby-distance process.

Before we proceed further, we should recall that in diploid and sexually reproducing plants, biparentally inherited DNA is typically represented by nuclear DNA (nuDNA), whereas paternally inherited DNA is represented (if at all) by either mtDNA and/or cpDNA, depending on the taxonomic group (Rebould and Zeyl, 1994; Mogensen, 1996). This implies a difference in ploidy between paternally and biparentally inherited DNA that needs to be taken into account in our considerations. However, because differences in ploidy levels are typically constant for a given species, at best the ploidy level should be 'cancelled' out to avoid undesired (constant) confounding effects on the impact of demographic and dispersal parameters on the relative SGS intensity. Hence, we focussed on the relatedness coefficient that does not depend on a ploidy level (that is, a ploidy level does not enter into the expected value of relatedness, see Equation (10) in Hardy and Vekemans, 1999) and can be directly used to compare SGS in diploid nuclear and haploid cytoplasmic genomes (Hardy and Vekemans, 2001). For this reason, the following results are specific to the relatedness coefficient and do not hold for the kinship coefficient that is routinely used in empirical studies.

If a population evolves according to the pure isolation-by-distance process, the slope of the relatedness correlogram has the theoretical expectation of

$$
b=-\frac{(1-F)}{(1+(k-1) F) 2 \pi D_{\mathrm{e}} \sigma^{2}}
$$

where $D_{\mathrm{e}}$ is the effective density of a population, $\sigma^{2}$ is the axial variance of gene dispersal, $F$ is the inbreeding coefficient and $k$ is the ploidy level (Hardy and Vekemans, 1999; Equation 15 therein). Nevertheless, because of absence of selfing, in dioecious plants inbreeding tends to be apparently less pronounced compared with monoecious species. We used this argument to simplify our considerations through assuming that $F=0$. Consequently, we obtained

$$
b \approx-\frac{1}{2 \pi D_{\mathrm{e}} \sigma^{2}}
$$

Nevertheless, as the assumption of $F=0$ may be violated because of biparental inbreeding, especially under limited dispersal, Equation (2) should be treated as a first approximation of the actual slope.

Following Vekemans and Hardy (2004), we assumed that $D_{\mathrm{e}}=D N_{\mathrm{e}} / N$, where $N$ and $N_{\mathrm{e}}$ are the census and effective population size, respectively. Consequently, in dioecious plants $D_{\mathrm{e}}$ can be represented by a function of the census population density $(D)$ and the (effective) proportion of sexes. We let $r$ be the proportion of effectively reproducing males. In the case of biparentally inherited DNA, $D_{\mathrm{e}(\mathrm{bi})}=4 r(1-r) D$. For paternally inherited DNA, $D_{\mathrm{e}(\text { pat })}=r D$.

Plants disperse their genes through seeds and pollen (except for maternally inherited genes that are dispersed through seeds only). Because half of the nuclear genes are dispersed through pollen (as haploid gametes) and the second half through seeds (as diploid zygotes), $\sigma_{\mathrm{bi}}^{2}=\frac{\sigma_{\mathrm{s}}^{2}}{2}+\frac{\left(\sigma_{\mathrm{s}}^{2}+\sigma_{\mathrm{p}}^{2}\right)}{2}=\sigma_{\mathrm{s}}^{2}+\sigma_{\mathrm{p}}^{2} / 2$, where 
$\sigma_{\mathrm{s}}^{2}$ and $\sigma_{\mathrm{p}}^{2}$ are the axial variances of gene dispersal for seeds and pollen, respectively (Crawford, 1984). For paternally transmitted DNA, $\sigma_{\mathrm{pat}}^{2}=\sigma_{\mathrm{s}}^{2}+\sigma_{\mathrm{p}}^{2}$, because all genes are dispersed first through pollen (from male to female) and then through seeds (from female to offspring). Using these quantities, one can predict that the ratio of the slopes of relatedness correlograms, reflecting the ratio of SGS intensities in paternally and biparentally inherited DNA, is

$$
\frac{b_{\mathrm{pat}}}{b_{\mathrm{bi}}}=4(1-r) \frac{\sigma_{\mathrm{s}}^{2}+\frac{\sigma_{\mathrm{p}}^{2}}{2}}{\sigma_{\mathrm{s}}^{2}+\sigma_{\mathrm{p}}^{2}}
$$

or

$$
\frac{b_{\text {pat }}}{b_{\mathrm{bi}}}=2(1-r) \frac{2+x}{1+x},
$$

where

$$
x=\frac{\sigma_{\mathrm{p}}^{2}}{\sigma_{\mathrm{s}}^{2}} .
$$

Thus, given that our approximate derivation holds true, one can predict that for equal sex proportions $(r=1 / 2)$ SGS tends to be more intense for paternally than biparentally inherited DNA, unless pollen dispersal is apparently more intense compared with seed dispersal (that is, $x$ becomes very large), when the difference in intensity vanishes (or the ratio goes to unity). When females prevail (that is, $r<1 / 2$ ), SGS in paternally inherited DNA is always more intense than SGS in biparentally inherited DNA (that is, $\frac{b_{\text {pat }}}{b_{\text {ti }}}>1$ ). On the other hand, for $r>3 / 4$, SGS in paternally inherited DNA is always less intense than SGS in biparentally inherited DNA (that is, $\frac{b_{\text {pat }}}{b_{b i}}<1$ ). Finally, for $1 / 2<r<3 / 4$, SGS in paternally inherited DNA is more intense than SGS in biparentally inherited DNA when $x<\frac{4 r-3}{1-2 r}$ and less intense otherwise.

\section{Simulation study}

We used computer simulations in order to verify the theoretical approximation of the relative strength of SGS in biparentally and paternally inherited genes, derived in the previous section. We assumed that diploid nuDNA represents a biparentally inherited genome, whereas (haploid) mtDNA represents a paternally inherited genome.

We assumed a completely isolated dioecious population of a constant size of 500 individuals and density of 20 individuals per ha. However, our conclusions should be robust to assumptions about population size and density (see Hardy and Vekemans, 1999). Furthermore, we assumed non-overlapping generations, no mutation and a constant dispersal process. In order to reveal the impact of sex proportions, we simulated populations with constant proportion of males $(15,30,50,70$ and $85 \%)$. Thus, our simulations did not take into account any variation of sex ratios in time, for example, due to demographic stochasticity (Kéry et al., 2003). To simulate dispersal, we assumed a simple exponential dispersal kernel that can be considered as the intermediate distribution between thin- and fat-tailed dispersal distributions in terms of mixing of propagules between distant locations (Klein et al., 2006). In order to study the impact of dispersal of seeds and pollen, we simulated a series of nine different dispersal patterns (Table 1).

The initial generation was created as follows. First, coordinates were drawn randomly within a square to obtain a density of 20 individuals per ha. Then, each individual was assigned sex and genotypes at a nuclear and mitochondrial locus, each with 10 equally frequent alleles. Nuclear genotypes followed Hardy-Weinberg proportions. Sex was assigned randomly according to the expected sex proportions.

As in the work of Leblois et al. (2004), parentages of every subsequent generation were generated through tracing the dispersal process back compared with the real dispersal process (that is, based on the so-called 'backward dispersal kernel'). In this approach, a position of the parental gene is determined based on the position of the descendant gene. Initially, 500 coordinates were drawn randomly within a population area. They represented the spatial distribution of newly regenerated progeny. Then, for every progeny, the mother was drawn randomly, according to the assumption of the exponential dispersal kernel. For the $i$-th progeny, the probability that the $j$-th adult is a mother (seed parent) equals

$$
\psi_{i j}=\frac{\theta_{j} \exp \left(-\frac{\sqrt{\left(x_{i}-x_{j}\right)^{2}+\left(y_{i}-y_{j}\right)^{2}}}{a_{s}}\right)}{\sum_{k=1}^{500} \theta_{k} \exp \left(-\frac{\sqrt{\left(x_{i}-x_{k}\right)^{2}+\left(y_{i}-y_{k}\right)^{2}}}{a_{s}}\right)},
$$

where $\left(x_{i}, y_{i}\right)$ and $\left(x_{j}, y_{j}\right)$ are the spatial coordinates for the $i$-th offspring and the $j$-th adult, respectively, $\theta_{j}$ is the binary indicator equal to 1 if the $j$-th individual is a female and 0 otherwise and $a_{s}$ is the scale of the exponential dispersal kernel for seeds. The scale $a$ of the exponential dispersal kernel is proportional to the forward average dispersal distance $\delta(\delta=2 a)$ (Austerlitz et al., 2004). Once the mother was known, the father was drawn randomly, based on the probability distribution obtained as previously, where for the $j$-th adult (mother) the probability that the $k$-th adult is a father (pollen donor) equals

$$
\varphi_{j k}=\frac{\left(1-\theta_{k}\right) \exp \left(-\frac{\sqrt{\left(x_{j}-x_{k}\right)^{2}+\left(y_{j}-y_{k}\right)^{2}}}{a_{p}}\right)}{\sum_{l=1}^{500}\left(1-\theta_{l}\right) \exp \left(-\frac{\sqrt{\left(x_{j}-x_{l}\right)^{2}+\left(y_{j}-y_{l}\right)^{2}}}{a_{p}}\right)},
$$

where $\left(x_{j}, y_{j}\right)$ and $\left(x_{k}, y_{k}\right)$ are the spatial coordinates for the $j$-th and the $k$-th adults, respectively, $\theta_{k}=1$ if the $k$-th adult was a female and 0 otherwise and $a_{p}$ is the scale of the exponential dispersal kernel for pollen. Once parents were drawn, offspring genotypes of nuclear and mitochondrial loci were randomly generated, following the assumption of biparental (Mendelian) inheritance of nuDNA and complete parental inheritance of mtDNA. Finally, sex of the offspring was assigned randomly according to the expected sex proportions.

In order to quantify the SGS intensity, matrices of pairwise relatedness coefficients (using Moran's I; Hardy and Vekemans, 1999) and Euclidean distances were computed and used to estimate slopes of regression functions, with the natural logarithm of distance taken as an explanatory variable. Slopes were computed separately for nuDNA and mtDNA after 100 generations. In addition, we also scored the proportion of cases when $b_{\mathrm{mt}}<b_{\mathrm{nu}}$. In order to compute theoretical expectations for the slopes, empirical axial variances of distances between mother and offspring, father and offspring and mother and father were computed and averaged across generations and replicates (100 replicates per scenario). Simulations were implemented in GNU Fortran, and the code (sgssimul.f95) is freely available at http://www.ukw.edu.pl/ pracownicy/strona/igor_chybicki.

\section{The case study of T. baccata}

To illustrate the theoretical predictions, we used the case of T. baccata, a European gymnosperm tree. Earlier cytological studies suggested biparental inheritance of mtDNA in Taxus (Chesnoy, 1987; Pannel and Bell, 1988), although the authors underlined that because of close similarity of male and female mitochondria, there is a need for genetic marker-based confirmatory tests (Pannel and Bell, 1988). However, our preliminary observations showed that in this species, $\sim 95 \%$ of seeds that resulted from open pollination did not share mitochondrial genotypes (mitotypes) with their mother trees (IJ Chybicki, personal observation). Therefore, we formulated a hypothesis that mtDNA is predominantly paternally inherited and designed controlled crosses to assess the compatibility in mtDNA between parents and progeny and, consequently, to verify the hypothesis about the mode of mtDNA inheritance.

\section{Controlled crosses}

In order to track the transmission of the mitochondrial genome, we designed a controlled crossing experiment. First, 10 females and 10 males were sampled within a population at the Arboretum of Institute of Dendrology Polish Academy of Sciences (Kórnik, Poland). The sampled population had originated spontaneously from natural regeneration and appears to show morphological traits, ecological and genetic structure similar to that observed in wild populations of English yew (Iszkuło and Boratyński, 2005; IJ Chybicki et al., 
Table 1 Theoretical expectations and observed values of slopes of correlograms for biparentally and paternally inherited nuclear $\left(b_{\text {nu }}\right)$ and mitochondrial $\left(b_{\mathrm{mt}}\right)$ DNA, respectively, under different simulation scenarios

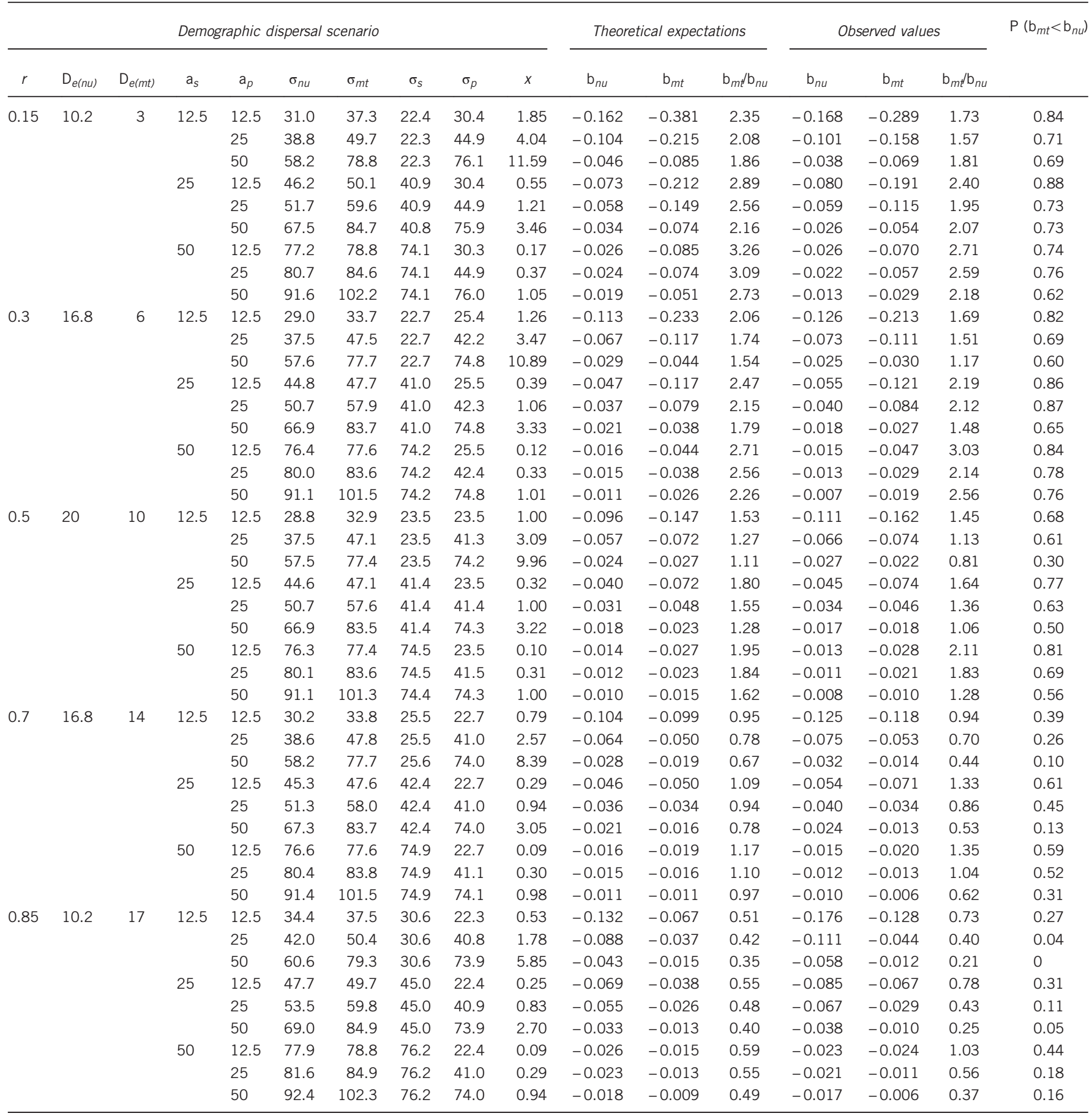

Abbreviations: $a_{\mathrm{s}}$ and $a_{\mathrm{p}}$, scale of the exponential dispersal kernel for seeds and pollen, respectively; $\sigma_{\mathrm{nu}}$ and $\sigma_{\mathrm{m} t}$, square root of axial variance of dispersal distance for nuclear and mitochondrial genes, respectively; $\sigma_{\mathrm{s}}$ and $\sigma_{\mathrm{p}}$, square root of axial variance of dispersal distance for seeds and pollen, respectively; $D_{\mathrm{e}(\mathrm{mt})}$, effective population density for mitochondrial genes; $D_{\mathrm{e}}$ (nu), effective population density for nuclear genes; $R$, proportion of males; $x=\sigma_{\mathrm{p}}^{2} / \sigma_{\mathrm{s}}^{2}, P\left(b_{\mathrm{mt}}<b_{\mathrm{nu}}\right)$, proportion of simulated data sets for which $b_{\mathrm{mt}}<b_{\mathrm{nu}}$.

unpublished results). Sampled males and females were subjected to DNA extraction and genotyped for the mitochondrial microsatellite marker TB01 (see DNA analysis). To optimise efforts, two males and four females were selected in order to arrange eight mating pairs, representing different mitotypic combinations (Table 2).

Further steps of the experiment were conducted as follows. First, macrostrobili (female reproductive organs) before their opening on four selected mother trees were isolated on 13 March 2013 with polyethylene bags in order to prevent undesirable pollination. Male branches were cut for pollen collection before their opening, put into water in the laboratory and stored at room temperature until flowering. Pollen from each father tree was harvested into a polyethylene bag. Pollen was applied to the bagged macrostrobili using a medicinal syringe on 11 April 2013. The bags from female branches were not removed until visible seeds appeared on 6 May 2013. In September 2013, all 
Table 2 Results of controlled crossing experiment designed to study transmission of mitochondrial DNA in Taxus baccata

\begin{tabular}{lccc}
\hline Female (mitotype) & Male (mitotype) & $\begin{array}{c}\text { Number of } \\
\text { embryos }\end{array}$ & $\begin{array}{c}\text { Number of paternal } \\
\text { mitotypes }\end{array}$ \\
\hline F2 (210) & M1 (165) & 28 & 28 \\
F3 (175) & 9 & 9 \\
F7 (210) & 10 & 10 \\
F9 (180) & 7 & 7 \\
F2 (210) & M2 (205) & 11 & 11 \\
F3 (175) & 4 & 3 \\
F7 (210) & 4 & 4 \\
F9 (180) & 8 & 8 \\
\hline
\end{tabular}

mature seeds were collected. The dormancy of $T$. baccata seeds was broken using a combination of warm and cold stratification according to the protocol described by Suszka (1985). Stratification lasted for 10 months, after which the germinated seeds were sown into plastic pots. The fully developed seedlings were harvested after 1 month and used for DNA extraction and mtDNA genotyping (see DNA analysis) as well as the assessment of mtDNA inheritance (see Statistical analyses).

\section{Natural populations}

To study SGS intensity in the mitochondrial vs nuclear genome, we used samples collected within two remnant populations of $T$. baccata well characterised for genetic structure in our previous studies (Chybicki et al., 2011, 2012). Within each population, sex proportions were estimated based on direct observations of reproductive organs or remains of seed envelopes attached to branches. The populations differ highly significantly with respect to census sex ratios $\left(\chi^{2}=68.76\right.$; d.f. $\left.=1 ; P<0.001\right)$ (Table 4). Furthermore, in statistical terms, 'Cisy w Czarnem' can be the representative of a population with equal census sex proportions because the binomial proportion of males does not deviate significantly from $0.5\left(\chi^{2}=0.006\right.$; d.f. $=1 ; P=0.940$, estimated based on a sample of 179 individuals; IJ Chybicki, personal observation). On the contrary, 'Cisy Staropolskie' represents a yew population with sex proportions significantly biased towards males (67\% males; estimated based on a sample of 242 individuals; see Iszkuło et al., 2009). It should be stressed that 'Cisy Staropolskie' seems to represent an exceptionally high proportion of males compared with the average sex proportions of the species (c. 50\%; see Iszkuło et al., 2009; Litkowiec et al., 2015). Male-biased sex proportions can be, at least partly, attributable to limited availability of water and stand density. Females of T. baccata tend to be more abundant in wetter habitat (Vessela et al., 2015). Our previous study showed that there is a positive correlation between average rainfall and increased participation of females in T. baccata populations (Iszkuło et al., 2009), and this may explain differences in sex proportions between Cisy Staropolskie and Cisy w Czarnem (the average annual rainfall of 572 and $638 \mathrm{~mm}$, respectively; based on DIVA-GIS software: Hijmans et al., 2005). The study populations also differ with respect to census density (Table 4 , see also Figure 1). According to the sex allocation theory, females are generally less competitive than males and females of English yew may have a higher mortality (Iszkuło et al., 2009). As shown in our earlier study, both populations reveal a significant SGS at six nuclear microsatellite markers (Chybicki et al., 2011) as well as relatively high genetic variation and no signal of a recent bottleneck (Chybicki et al., 2012). In addition, Cisy Staropolskie showed low (that is, $F=0.04$ ), but statistically significant inbreeding (Chybicki et al., 2011).

\section{DNA analysis}

$m t D N A$ analysis. Parental trees and seedlings germinated from seeds produced in controlled crossing experiment were subject to mtDNA genotyping. For this purpose, the total genomic DNA was extracted from needles taken from progeny and parents using the CTAB protocol (Doyle and Doyle, 1990). In the case of samples from natural populations, we used DNA extracted previously (see Chybicki et al., 2011) and stored at $-80^{\circ} \mathrm{C}$.
Within 'Cisy w Czarnem' and 'Cisy Staropolskie' we sampled a subset of 216 and 293 individuals, respectively.

As a molecular marker for the mitochondrial genome, we used the single pentanucleotide microsatellite locus TB01 developed for Taxus yunnanensis (Miao et al., 2008), based on the sequence published previously for Taxus brevifolia (Gene Bank accession: AB029370; Chaw et al., 2000). The original sequence is a fragment of the gene of $18 \mathrm{~S}$ rRNA. Based on the sequence for T. brevifolia, PCR primers were redesigned (using Primer 3; Koressaar and Remm, 2007) to obtain a PCR product between 135 and $220 \mathrm{bp}$ (forward primer: 5'-ATCTATTCGGGGCTCGATCT-3', reverse primer: 5'-ATCCCT CGCCCCCTAGATTA-3'). The forward primer was labelled with FAM fluorescent dye.

The total PCR mixture of $10 \mu \mathrm{l}$ contained 10 ng of total genomic DNA, $5 \mu \mathrm{l}$ of Taq PCR Master Mix (Qiagen, Hilden, Germany) and $50 \mathrm{~nm}$ of forward and reverse primers. The PCR reaction proceeded under the following conditions: $94^{\circ} \mathrm{C}$ for $3 \mathrm{~min} ; 10$ cycles of $94^{\circ} \mathrm{C}$ for $30 \mathrm{~s}, 65^{\circ} \mathrm{C}\left(-1{ }^{\circ} \mathrm{C}\right.$ per cycle $)$ for $45 \mathrm{~s}$ and $72{ }^{\circ} \mathrm{C}$ for $45 \mathrm{~s}$; and 25 cycles of $94{ }^{\circ} \mathrm{C}$ for $30 \mathrm{~s}, 55^{\circ} \mathrm{C}$ for $45 \mathrm{~s}$ and $72{ }^{\circ} \mathrm{C}$ for $45 \mathrm{~s}$. The final extension step at $72^{\circ} \mathrm{C}$ lasted for $10 \mathrm{~min}$. The PCR was conducted using a PTC200 thermal cycler (Bio-Rad, Hercules, CA, USA). PCR products were sized using an ABI PRISM 3130XL capillary sequencer (Applied Biosystems, Carlsbad, CA, USA) and post-processed using GeneScan 3.7 (Applied Biosystems) and Genotyper 3.7 software (Applied Biosystems). Thanks to clear separation of PCR products for a pentanucleotide microsatellite sequence, mitotypes were determined unequivocally based on the size of the PCR product.

nuDNA analysis. For the purpose of comparative assessment of spatial genetic structure, we used data on six microsatellite markers assayed previously for the two natural populations. Details are described in Chybicki et al. (2011).

\section{Statistical analyses}

Analysis of mtDNA inheritance. Because embryos obtained from controlled crossing had either paternal or maternal mitotype, the mode of inheritance of mtDNA was quantified directly through the probability of paternal transmission $(q)$. For this purpose, we used a Bayesian approach based on the observed number of embryos carrying paternal $\left(n_{\mathrm{p}}\right)$ and maternal $\left(n_{\mathrm{m}}\right)$ mitotypes. Because each pollination experiment was an independent replicate, $n_{\mathrm{p}}$ and $n_{\mathrm{m}}$ followed a binomial distribution, with $q$ taken as the probability of observing an embryo carrying a paternal mitotype. Consequently, the binomial distribution was used as a model (the likelihood function), with $q$ being an (estimable) parameter of interest. As a prior for $q$, we assumed a beta distribution with both shape parameters equal to 0.5 , corresponding to the so-called 'Jeffreys prior', known for yielding good interval estimates of proportions (Brown et al., 2001). We need to emphasise that the chosen prior distribution corresponds to $a$ priori probability of paternal transmission equal to 50\% (Chesnoy, 1987; Pannel and Bell, 1988). Thus, our approach reflects the results of cytological observations that mtDNA can be transmitted from the mother and the father with a high probability.

Genetic structure of natural populations. For natural populations, standard descriptors of genetic structure were computed, including number of alleles $(A)$, effective number of alleles $\left(A_{\mathrm{e}}\right)$ and genetic diversity $(D$, computed as heterozygosity expected under the Hardy-Weinberg equilibrium). Effective number of alleles was computed as $1 /(1-D)$. SGS was analysed using a spatial autocorrelogram based on the relatedness coefficient (Moran's I; Hardy and Vekemans, 1999) computed for both the mitochondrial microsatellite marker and six nuclear microsatellite markers assayed in the earlier study (Chybicki et al., 2011). Deviation of the slope from zero was verified based on 10000 permutations of coordinates. The intensity of SGS in mtDNA and nuDNA was assessed with the slope of regression function. SGS intensities were treated as significantly different if the value of the slope for mtDNA was outside the confidence interval obtained for the slope for nuDNA. The confidence interval was approximated as $2 \times$ s.e., where s.e. is the standard error computed using jackknifing over loci. Analyses were performed using SPAGeDi 1.5 (Hardy and Vekemans, 2002). 


\section{Cisy w Czarnem}

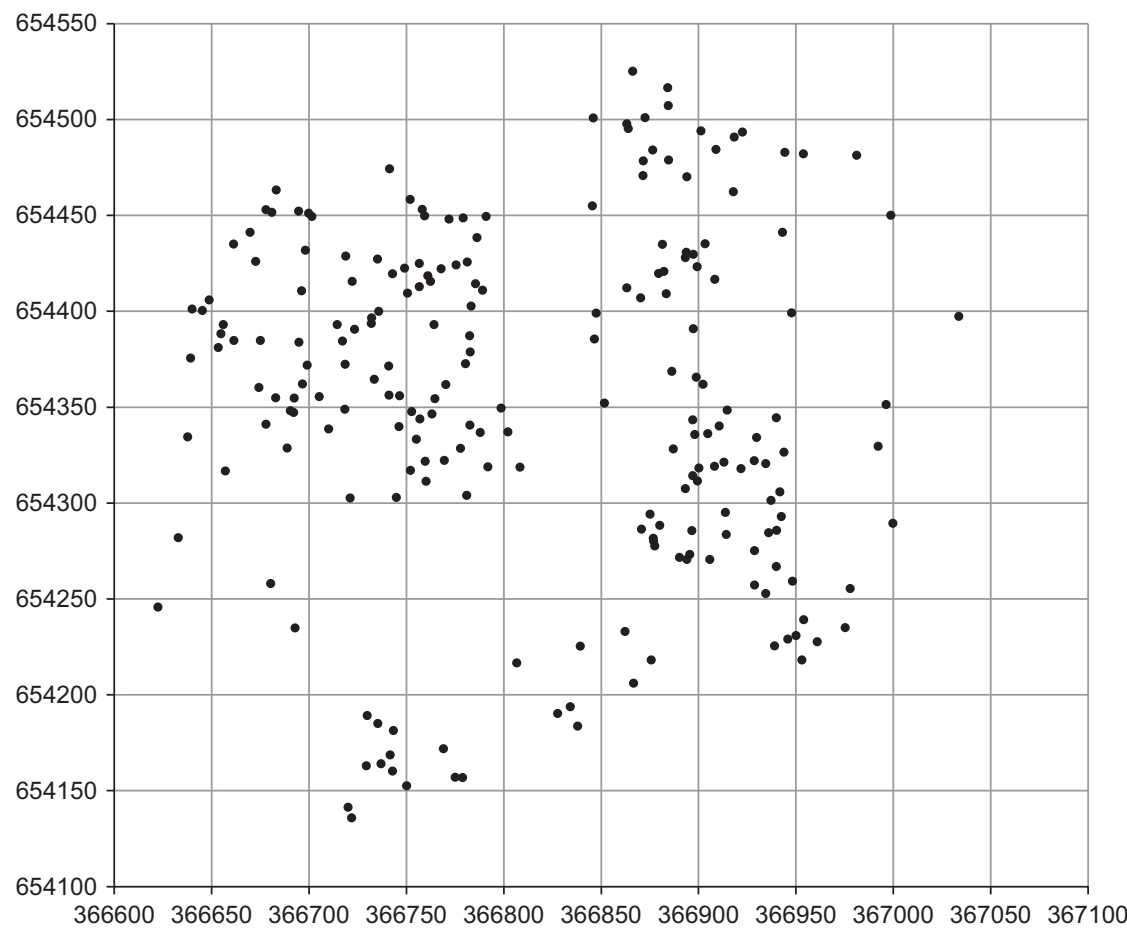

\section{Cisy Staropolskie}

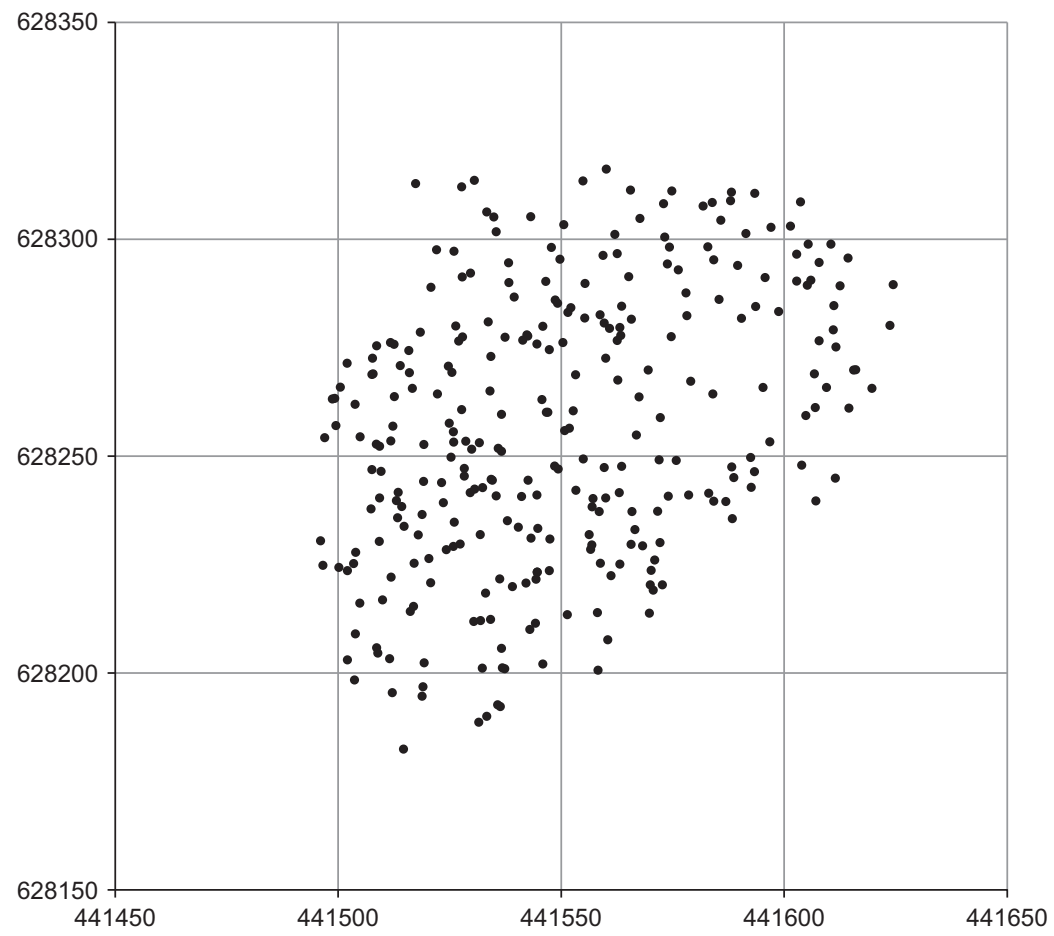

Figure 1 Distribution of individuals sampled within the study populations. The coordinates are given in metres (the ETRS89 system).

\section{RESULTS}

Simulation results

The slopes of regression function for relatedness on the logarithm of distance observed in the simulation study were generally in good accordance with the values expected under the approximate theory derived in this study (Figure 2). The average relative bias, computed as the difference between the expectation and the value observed in simulations divided by the expected value, was equal to 2.7 and $-5.7 \%$ for biparentally and paternally inherited DNA, respectively. In other words, the slope predicted by the approximate theory was slightly 
Biparentally inherited DNA

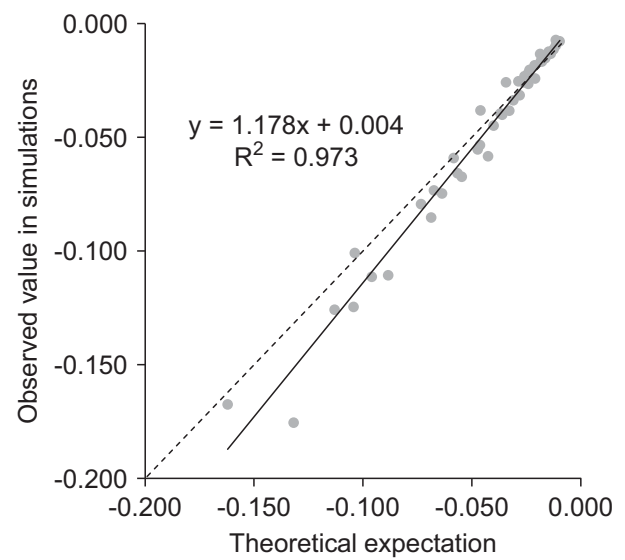

Paternally inherited DNA

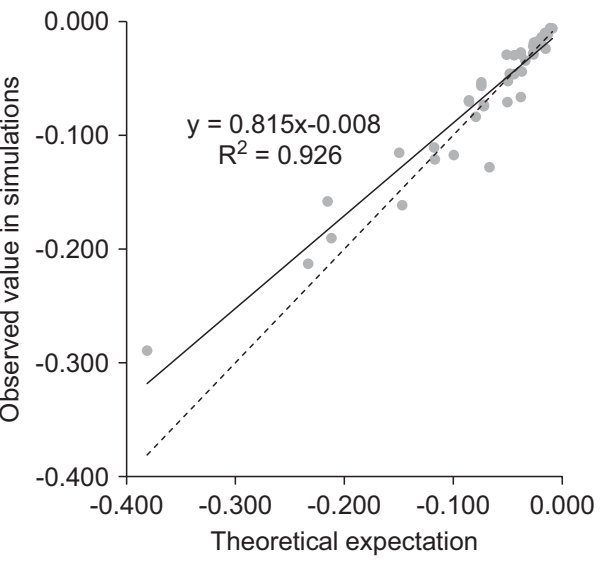

Figure 2 Results of the simulation study. Expected (dashed lines) and observed (dots) slopes of regression functions for relatedness vs the logarithm of distance for biparentally and paternally inherited DNA. Solid lines show the best-fitting linear function.

underestimated in the case of biparentally inherited DNA, whereas it was overestimated in the case of paternally inherited DNA when compared with the simulation results.

Generally, simulation results confirmed our theoretical predictions that the ratio of slopes $b_{\mathrm{mt}} / b_{\mathrm{nu}}$ tends to be greater than unity unless proportion of males to females exceeds $1 / 2$. In the scenarios of male deficiency $\left(r=0.15\right.$ and 0.3 ), the grand average for $b_{\mathrm{mt}} / b_{\mathrm{nu}}$ equaled 2.1, whereas the proportion of individual cases of $b_{\mathrm{mt}}<b_{\mathrm{nu}}$ was $75 \%$. It should be stressed that even if pollen dispersal was 10 times greater than seed dispersal, the proportion of cases where $b_{\mathrm{mt}}<b_{\mathrm{nu}}$ was $60 \%$.

In the case of equal sex proportions $(r=0.5), b_{\mathrm{mt}} / b_{\mathrm{nu}}>1$ for all dispersal scenarios, with the exception of a single case in which pollen dispersal was $\sim 10$ times greater than seed dispersal. The proportion of cases of where $b_{\mathrm{mt}}>b_{\mathrm{nu}}$ was generally $>0.5$, except for two dispersal scenarios, when pollen was 3 and 10 times greater than seed dispersal.

As expected, under sex proportions biased moderately towards males $(r=0.7), b_{\mathrm{mt}} / b_{\mathrm{nu}}$ considerably depended on the relative pollento-seed dispersal capacity. The $b_{\mathrm{mt}} / b_{\mathrm{nu}}$ ratio was greater than unity, and the proportion of cases where $b_{\mathrm{mt}}<b_{\mathrm{nu}}$ was $>50 \%$ when pollen dispersal was at least 3 times lower than seed dispersal. Finally, under apparent excess of males $(r=0.85)$, we observed $b_{\mathrm{mt}} / b_{\mathrm{nu}}>1$ for no single scenario. In that case, the grand average for $b_{\mathrm{mt}} / b_{\mathrm{nu}}$ equalled 0.17 .

Finally, we performed a multiple logistic regression analysis for the proportion of cases of $b_{\mathrm{mt}}<b_{\mathrm{nu}}$ as a response variable and pollen-toseed dispersal ratio $(x)$, the proportion of males $(r)$ and their interaction $(x \times r)$ taken as explanatory variables. The analysis revealed that the proportion of $b_{\mathrm{mt}}<b_{\mathrm{nu}}$ depends negatively on $r(t=-5.76$; $P<0.001)$, and the interaction $x \times r(t=-4.04 ; P<0.001)$ whereas not on $x$ as a separate factor $(t=1.18 ; P=0.245)$.

\section{The case study of $T$. baccata}

Transmission of mtDNA. Among 10 males and 10 females initially sampled as potential parents, we found two and five mitotypes, respectively (Table 2 ). Based on 8 incompatible pairs, 81 seeds were successfully harvested, germinated and genotyped using TB01, an mtDNA microsatellite marker. In all, 80 seedlings (98.8\%) revealed a paternal mitotype, whereas a single seedling revealed a maternal mitotype (Table 2). The Bayesian posterior mode, median and mean of the probability of paternal transmission $(q)$ were equal to 0.994 ,
Table 3 The summary of genetic variation of Taxus baccata populations at 1 mitochondrial DNA (mtDNA) and 6 nuclear DNA (nuDNA) microsatellites

\begin{tabular}{lcc}
\hline Population & Cisy w Czarnem & Cisy Staroploskie \\
\hline$m t D N A$ & & \\
Size range (bp) & $145-210$ & $135-205$ \\
$A$ & 13 & 9 \\
$A_{\mathrm{e}}$ & 4.02 & 2.98 \\
$D$ & 0.751 & 0.665 \\
$n u D N A^{\text {a }}$ & & \\
$A$ & 23.5 & 17.7 \\
$A_{\mathrm{e}}$ & 8.1 & 6.0 \\
$D$ & 0.871 & 0.814 \\
\hline
\end{tabular}

Abbreviations: $A$, number of mitotypes (alleles in nuDNA); $A_{\mathrm{e}}$, effective number of mitotypes (alleles in nuDNA); D, genetic diversity (expected heterozygosity in nuDNA); Size range, the observed range for PCR products at the mitochondrial microsatellite marker.

In the case of nuDNA, averages over six microsatellite markers are shown.

aData from Chybicki et al. (2011).

0.985 and 0.982 , respectively. The $95 \%$ equal-tail credible interval ranged from 0.944 to 0.999 .

Genetic structure of natural populations. Both natural populations revealed polymorphism at the mitochondrial microsatellite locus (Table 3). The numbers of haplotypes equalled 13 and 9 in Cisy $w$ Czarnem and Cisy Staroploskie, respectively. However, because of uneven distribution of mitotype frequencies, the effective numbers of alleles were much lower and equalled $\sim 4$ and 3 , respectively. In addition, Cisy w Czarnem and Cisy Staropolskie were characterised by diversity equal to 0.751 and 0.665 , respectively.

Based on the permutation test, mitotypes showed nonrandom SGS in Cisy w Czarnem in the first distance class (up to $34 \mathrm{~m}$ ) (Figure 3). On the other hand, no deviation from complete spatial randomness in mtDNA was observed in Cisy Staroploskie. Slopes of the regression function for the logarithm of distance vs relatedness were -0.018 and -0.004 in Cisy $\mathrm{w}$ Czarnem and Cisy Staroploskie, respectively (Table 4). Nonetheless, only the former value deviated significantly from zero (complete spatial randomness), as revealed by the $95 \%$ confidence interval generated after permuting individual coordinates. 

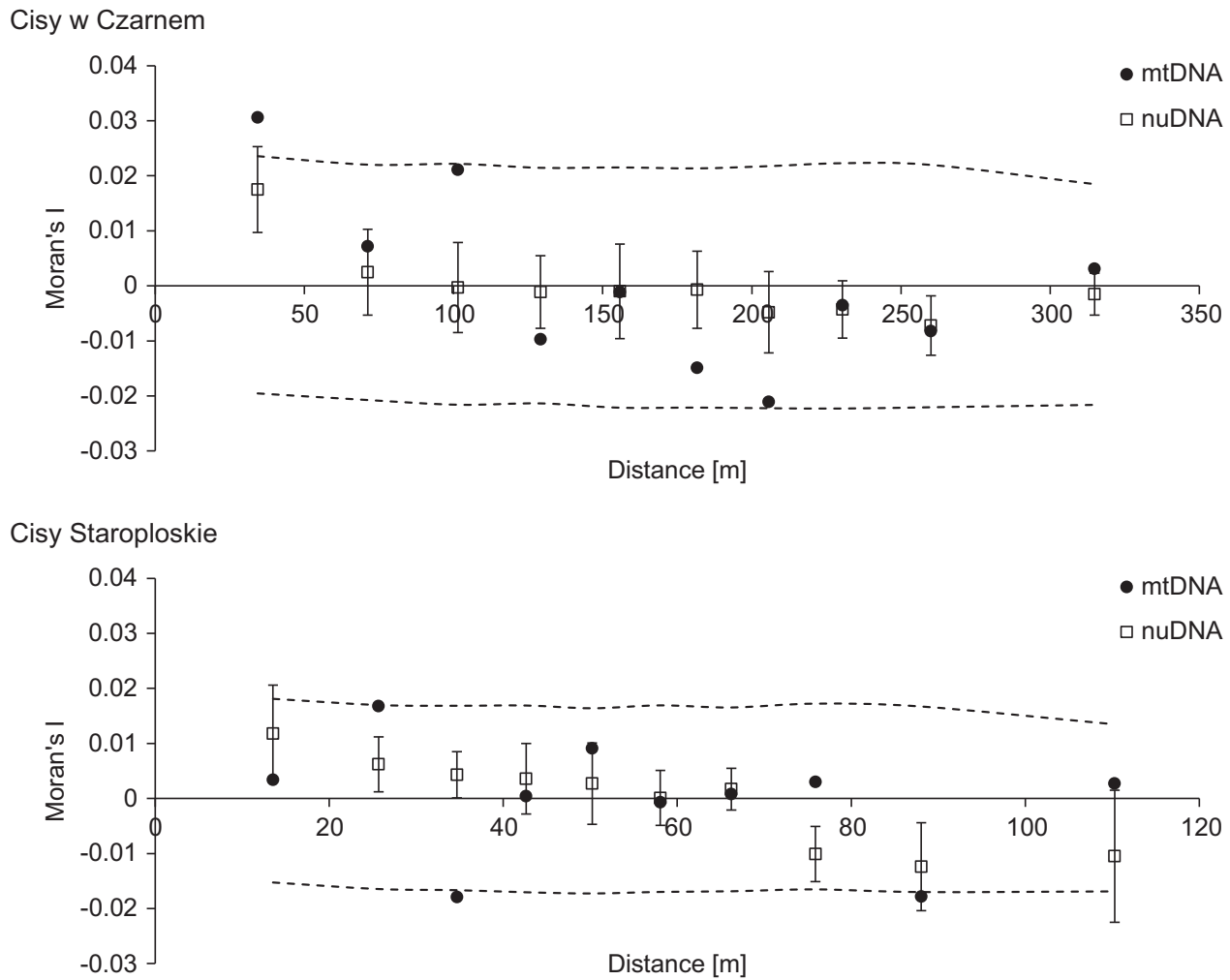

Figure 3 Relationship between pairwise relatedness in mtDNA or nuDNA and distance observed in Taxus baccata populations. Bars show $95 \%$ confidence intervals. Dashed lines show limits of $95 \%$ confidence interval around the null hypothesis of complete spatial randomness in mtDNA.

Table 4 Spatial genetic structure of Taxus baccata populations at 1 mitochondrial DNA (mtDNA) and 6 nuclear DNA (nuDNA) microsatellites

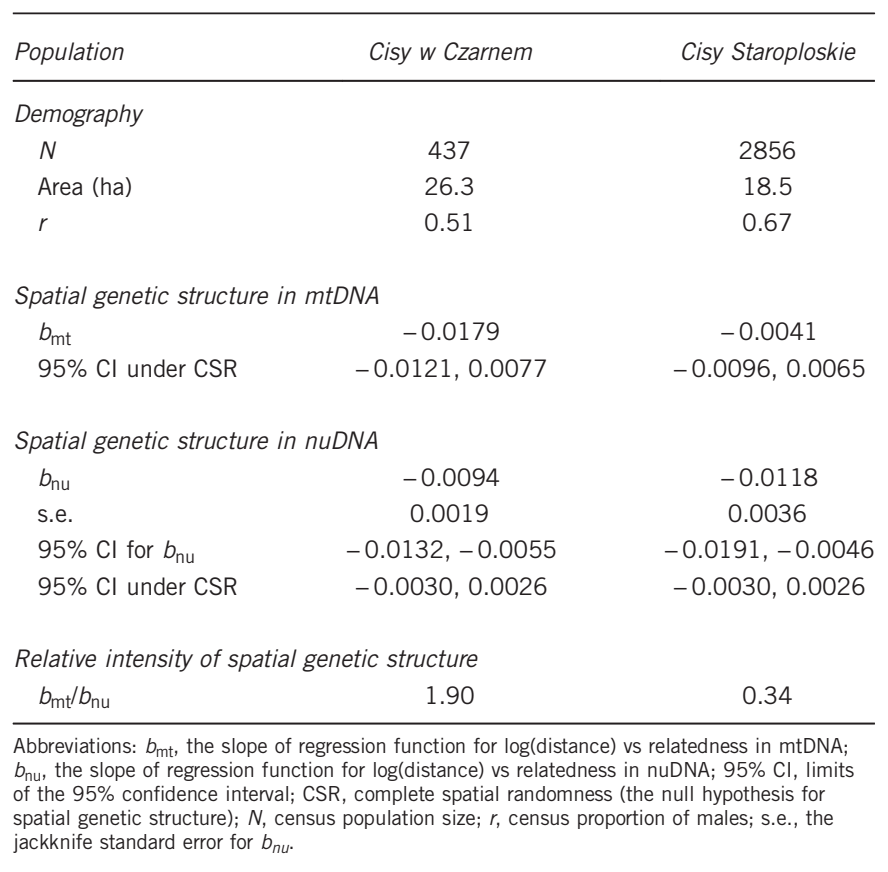

In the case of nuclear microsatellites, the slopes of regression function were -0.009 and -0.012 in Cisy w Czarnem and Cisy Staroploskie, respectively. Both slope values deviated significantly from zero, but they did not differ significantly from each other, as the $95 \%$ confidence interval around the slope for a given population did not include the estimate for the other population. In both populations, the slope for mtDNA was outside the $95 \%$ confidence intervals obtained for nuDNA. However, mtDNA revealed higher and lower SGS intensity compared with nuDNA in Cisy w Czarnem and Cisy Staropolskie, respectively. The ratio of slopes equalled 1.9 and 0.34 in Cisy w Czarnem and Cisy Staropolskie, respectively.

\section{DISCUSSION}

This study provides theoretical arguments for the prediction that in a dioecious plant the relative strength of fine-scale SGS in paternally and biparentally inherited DNA depends on both sex proportions and the interaction of sex proportions and the pollen-to-seed dispersal ratio. The effective population density assessed for paternally inherited DNA depends on the proportion of males in a population but, as we showed in the theoretical derivations, typically is much lower compared with the population density for biparentally inherited nuclear genes. On the other hand, dispersal is generally larger for paternally inherited genes, except in situations when the pollen-to-seed dispersal ratio approaches zero (Hamilton and Miller, 2002; and this study). However, both the theoretical approximation and the simulation study showed that the compound impact of dispersal and effective population density leads to the development of more intense SGS in paternally inherited DNA under most circumstances. Only strongly male-biased dioecious populations are expected to reveal the opposite pattern of the relative SGS intensity.

\section{Theoretical results}

The simulation results were generally in good agreement with what we predicted based on the theoretical derivations. However, we observed some discrepancies that, in our opinion, resulted potentially from 
differences in assumptions made for the theoretical and empirical models as well as some simplifications. One of the most obvious differences was that in the case of theoretical derivations, we neglected the impact of inbreeding that can accumulate in simulated populations. In theory, the effect of inbreeding can be predicted based on the slopes expected under the pure isolation-by-distance model (Hardy and Vekemans, 1999). Because we considered the ratio of slopes, the term $(1-F)$ in the numerator of Equation (1) cancels out. However, in the case of biparentally inherited DNA, inbreeding incorporates to the denominator of the expected slope (Equation (1)). Consequently, the expected ratio $b_{\mathrm{mt}} / b_{\mathrm{nu}}$ as predicted by Equation (3) is slightly underestimated (the bias equals to $F \times b_{\mathrm{mt}} / b_{\mathrm{nu}}$ ). The bias is particularly expected in the case of limited gene dispersal, when inbreeding can result from mating between relatives. Using our simulation results, we attempted to verify whether the observed bias in $b_{\mathrm{mt}} / b_{\mathrm{nu}}$ is attributable to dispersal scenario. For this purpose, we took values of $b_{\mathrm{mt}} / b_{\mathrm{nu}}$ obtained in simulations (see Table 1, section 'Observed values') as empirical estimates of the expected values. These values were compared with the theoretical predictions estimated based on Equation (3). The deviation of the theoretical prediction from the empirical expectation was used as a dependent variable, whereas the parameter of total gene dispersal $\left(\sigma_{\text {nu }}\right)$ was used as an explanatory variable. Regression analysis revealed, however, that the deviation is not associated with gene dispersal $\left(\sigma_{\mathrm{nu}}\right)$ ( $t$ for the slope equalled to $0.368 ; P=0.714$ ), suggesting that any inconsistency between the theoretical approximation and simulation results is not specifically due to increased inbreeding.

Another assumption made in the theoretical derivations was that $D_{\mathrm{e}} / D=N_{\mathrm{e}} / N$. This assumption seems reasonable, and some unpublished results suggest it should be appropriate as long as the density is constant in space (Vekemans and Hardy, 2004). Our simulation model assumed random spatial distribution and constant population density. We also assumed that there were no differences in fecundity among plants. Therefore, there is no obvious reason to expect that the assumption made for theoretical derivations regarding the effective density can bias the results. Another source of bias can be the time needed to approach the stationary SGS. In all simulations, we assumed that 100 generations were sufficient for the development of stationary SGS. However, earlier simulations showed that the time to equilibrium increases as dispersal capacity decreases and can be as high as several hundred generations (Hardy and Vekemans, 1999). Consequently, some results observed in the simulation may not represent the theoretical equilibrium. Because histories of natural populations rarely trace back beyond a hundred generations, especially in dioecious gymnosperms, which are often represented by long-lived trees, we believe that our simulations quite well reflect the reality.

Although in some gymnosperms sex can be determined genetically (Lee, 1954), the environmental impact is often suggested to play a dominant role in sex determination for some species (Bierzychudek and Eckhart, 1988). Moreover, in dioecious plants, the sexes can experience somewhat independent processes of natural selection and, consequently, sex-specific ecological optima (Bierzychudek and Eckhart, 1988). Therefore, the distribution of males and females can show a level of spatial aggregation (Gao et al., 2012) that, in turn, can reflect environmental heterogeneity (Eppley, 2005). Nonrandom distribution of the sexes in space has a strong impact on the effective mating distance (the backward pollen dispersal kernel). Our theoretical considerations did not directly address this issue. However, because the backward dispersal kernel is the actual factor of both the theoretical and simulated SGS, our conclusions are valid for experimental data, regardless of circumstances determining the backward dispersal kernel in natural conditions.

In our simulations, the process of seed and pollen dispersal was assumed to follow a simple exponential distribution. As mentioned in the Materials and methods section, the exponential distribution represents an intermediate case in respect to the fatness of the tail (that is, the shape) of dispersal function. However, it may represent an unrealistic 'average' dispersal scenario. In many natural populations, seeds and pollen dispersal can follow thin-tailed (for example, because of barochory; Clark et al., 1999) or fat-tailed dispersal kernels (for example, because of anemophily; Austerlitz et al., 2004). Fortunately, the isolation-by-distance process appeared to be almost insensitive to the shape of dispersal kernel (Furstenau and Cartwright, 2016), making our conclusions independent of the assumed dispersal distribution.

\section{The case study of $T$. baccata}

In this study, we used T. baccata as the case study species. Analysis of the two natural populations revealed differences in the relative intensity of SGS in mtDNA vs nuDNA that can be attributable to differences in sex proportions. To this extent, our empirical results were in line with the theoretical predictions. It is noteworthy that the empirical results pinpoint a likely dispersal scenario. The ratio of slopes $b_{\mathrm{mt}} / b_{\mathrm{nu}}$ equalled 1.9 in Cisy w Czarnem. Assuming that the effective sex ratio does not deviate from 0.5 , such a high value can be observed only if pollen dispersal is less intensive than seed dispersal (Table 1). This could be the case in the studied species, as suggested elsewhere (Chybicki et al., 2011), because fleshly fruits are quite effectively dispersed by birds (Iszkuło and Boratyński, 2005), whereas wind-borne pollen has no specific facilities (such as specific structures observed in, for example, Pinaceae) or opportunities (such as a high elevation of the release point) to travel very long distances. Nonetheless, the results for Cisy Staropolskie seem to suggest the opposite (that is, that pollen and seed dispersal capacities are comparable). In addition, the ratio of slopes as low as 0.34 observed for Cisy Staropolskie would require sex proportions to be even more biased towards males than the census male proportion of the population. The two populations investigated in the case study differ significantly with respect to population density and light conditions. Because density has a negative impact on a natural regeneration in yew populations (Vessella et al., 2015) whereas light availability can influence female fecundity (Saniga, 2000), it is likely that different ecological processes can shape quite different backward dispersal kernels and different effective sex proportions in these populations.

Because the two study populations differ statistically neither in the SGS intensity in nuclear genes (see Table 4) nor in inbreeding levels (Chybicki et al., 2011), any confounding effect of inbreeding contributing to differences in $b_{\mathrm{mt}} / b_{\mathrm{nu}}$ ratio can be neglected. We cannot exclude, however, that the other confounding factors, such as colonisation history or past demography, contributed to the observed SGS patterns. Our previous study showed, for example, that Cisy Staropolskie are characterised by apparently lower ratio of effective $\left(N_{\mathrm{e}}\right)$ to census population size $(N)$ than Cisy w Czarnem $\left(N_{\mathrm{e}} / N=0.033\right.$ vs 0.40 , respectively; Chybicki et al., 2012) that could not be explained simply by differences in sex proportions. In addition, population histories, including possible variation of sex ratios, remain unknown except for the genetic evidence of no recent bottleneck (Chybicki et al., 2012). Thus, although the observed SGS patterns seem to reflect differences in sex proportions between the populations, we postulate to treat the conclusions with caution. 


\section{Transmission of mtDNA in Taxus}

Our study showed that the mitochondrial genome is predominantly paternally inherited in T. baccata. To some extent, our result disagrees with the direct cytological observations for Taxus species that suggest that mtDNA is biparentally transmitted (Chesnoy, 1987; Pannel and Bell, 1988). However, data on molecular variation in T. yunnanensis, based on the same mitochondrial marker that we used for T. baccata, showed absolute homozygosity in the surveyed individuals, despite the moderate level of genetic diversity at the locus (Miao et al., 2008). The absence of heterozygous (heteroplasmic) individuals noted in the study of T. yunnanensis (Miao et al., 2008) suggests uniparental inheritance of the mtDNA in Taxus and, as such, is concordant with observations made in our study. Nonetheless, because we observed a single case of maternal transmission of mtDNA, we do consider mtDNA as being generally biparentally inherited, although with extremely uneven contribution of the two sexes. Such a pattern can be a result of the bias in the initial number of copies of mtDNA derived from the mother and the father. Because a number of copies of mtDNA per cell is finite, the transmission of mtDNA during ontogeny resembles the process of random drift (Birky et al., 1978). As a result, despite the initial presence of two mitotypes in a zygote, a single mitotype is eventually fixed within a single cell after many generations of cell division. The theory of genetic drift predicts that the fixation probability is equal to the initial allele frequency. Consequently, we can expect that a mitotype present in a zygote at a higher proportion should most often be subject to fixation. One implication of this assumption is that any disproportionality in frequency between maternal and paternal mitotypes should most often lead to the fixation of the prevailing mitotype at later ontogenetic stages. Furthermore, there should be rare cases of the fixation of a minor mitotype. Heteroplasmic or mosaic individuals (that is, possessing both maternal and paternal mitotypes in the same cell or in different cells) should also be observed, although in a very low proportion. In addition, the level of heteroplasmy should decrease rapidly together with age of an individual. Detailed studies are needed, however, to reveal the potential to produce heteroplasmy in wild yew populations. MACPR (microsatellite DNA allele counting based on peak ratios; Esselink et al., 2004) or similar quantitative techniques can be used for this purpose. Adopting the theory of genetic drift to the results of controlled crossing enabled us to predict that the contribution of maternal mtDNA to a zygote should not exceed $\sim 5 \%$, but could be as low as $<0.1 \%$.

\section{CONCLUSIONS}

Comparative assessment of genetic structure at markers differing in the mode of inheritance has long been suggested as an effective tool for inferences about plant dispersal strategies (Ennos, 1994; Hamilton and Miller, 2002). However, our theoretical work supported by the case study of natural populations showed that nuclear and paternally inherited cytoplasmic DNA markers can improve our understanding of seed and pollen dispersal as well as effective sex ratios in real dioecious populations not only through the analysis of genetic differentiation among populations (Hamilton and Miller, 2002), but also through fine-scale SGS.

\section{DATA ARCHIVING}

Genotype data and coordinates available from the Dryad Digital Repository: http://dx.doi.org/10.5061/dryad.ff708.

\section{CONFLICT OF INTEREST}

The authors declare no conflict of interest.

\section{ACKNOWLEDGEMENTS}

We thank Olivier Hardy for his comments on the theoretical derivations as well as the editor and three anonymous reviewers for their valuable suggestions to improve the manuscript. This study was supported by several funds. DNA samples from natural populations were collected within the Polish National Science Centre (NCN) project NN304 421633 to IJC. The controlled crossing experiment and seed stratification were conducted thanks to the statutory funding of the Institute of Dendrology, Polish Academy of Sciences and Faculty of Biological Sciences, University of Zielona Góra. Simulations, mtDNA genotyping and data analyses were performed within the NCN project UMO-2014/15/B/NZ9/04404 to IJC.

Austerlitz F, Dick CW, Dutech C, Klein EK, Oddou-Muratorio S, Smouse P et al. (2004). Using genetic markers to estimate the pollen dispersal curve. Mol Ecol 13: 937-954.

Barluenga M, Austerlitz F, Elzinga JA, Teixeira S, Goudet J, Bernasconi G (2011). Fine-scale spatial genetic structure and gene dispersal in Silene latifolia. Heredity 106: 13-24.

Bierzychudek P, Eckhart V (1988). Spatial segregation of the sexes of dioecious plants. Am Nat 132: 34-43.

Birky Jr CW, Demko CA, Perlman PS, Strausberg RL (1978). Uniparental inheritance of mitochondrial genes in yeast: dependence on input bias of mitochondrial DNA and preliminary investigations of the mechanism. Genetics 89: 615-651.

Brown LD, Cai TT, DasGupta A (2001). Interval estimation for a binomial proportion. Stat Sci 16: 101-133.

Chaw SM, Parkinson CL, Cheng Y, Vincent TM, Palmer JD (2000). Seed plant phylogeny inferred from all three plant genomes: monophyly of extant gymnosperms and origin of Gnetales from conifers. Proc Natl Acad Sci USA 97: 4086-4091.

Chesnoy L (1987). La reproduction sexuée des Gymnospermes. Bull Soc Bot Fr Actual Bot 134: 63-85.

Chybicki IJ, Oleksa A, Burczyk J (2011). Increased inbreeding and strong kinship structure in Taxus baccata estimated from both AFLP and SSR data. Heredity 107: 589-600.

Chybicki IJ, Oleksa A, Kowalkowska K (2012). Variable rates of random genetic drift in protected populations of English yew: implications for gene pool conservation. Conserv Genet 13: 899-911.

Clark JS, Silman M, Kern R, Macklin E, HillRisLambers J (1999). Seed dispersal near and far: patterns across temperate and tropical forests. Ecology 80: 1475-1494.

Crawford TJ (1984). The estimation of neighbourhood parameters for plant populations. Heredity 52: 273-283.

Doyle J, Doyle J (1990). Isolation of plant DNA from fresh tissue. Focus 12: 13-15.

Ennos RA (1994). Estimating the relative rates of pollen and seed migration among plant populations. Heredity 72: 250-259.

Eppley SM (2005). Spatial segregation of the sexes and nutrients affect reproductive success in a dioecious wind-pollinated grass. Plant Ecol 181: 179-190.

Esselink GD, Nybom H, Vosman B (2004). Assignment of allelic configuration in polyploids using the MAC-PR (microsatellite DNA allele counting-peak ratios) method. Theor Appl Genet 109: 402-408.

Field DL, Pickup M, Barret SCH (2013). Comparative analyses of sex-ratio variation in dioecious flowering plants. Evolution 67: 661-672.

Furstenau TB, Cartwright RA (2016). The effect of the dispersal kernel on isolation-bydistance in a continuous population. PeerJ 4: e1848.

Gao J, Queenborough SA, Chai JP (2012). Flowering sex ratios and spatial distribution of dioecious trees in a south-east asian seasonal tropical forest. J Trop For Sci 24: 517-527

Givnish TJ (1980). Ecological constraints on the evolution of breeding systems in seed plants: dioecy and dispersal in gymnosperms. Evolution 34: 959-972.

Hamilton MB, Miller JR (2002). Comparing relative rates of pollen and seed gene flow in the island model using nuclear and organelle measures of population structure. Genetics 162: 1897-1909.

Hardy OJ, Vekemans X (2002). SPAGeDi: a versatile computer program to analyse spatial genetic structure at the individual or population levels. Mol Ecol Notes 2: 618-620.

Hardy OJ, Vekemans X (2001). Patterns of allozyme variation in diploid and tetraploid Centaurea jacea at different spatial scales. Evolution 55: 943-954.

Hardy OJ, Vekemans X (1999). Isolation by distance in a continuous population: reconciliation between spatial autocorrelation analysis and population genetics models. Heredity 83: 145-154.

Hijmans RJ, Cameron SE, Parra JL, Jones PG, Jarvis A. (2005). Very high resolution interpolated climate surfaces for global land areas. Int J Climatol 25: 1965-1978.

Iszkuło G, Boratyński A (2005). Different age and spatial structure of two spontaneous subpopulations of Taxus baccata as a result of various intensity of colonization process. Flora 200: 195-206.

Iszkuło G, Jasińska AK, Giertych MJ, Boratyński A (2009). Do secondary sexual dimorphism and female intolerance to drought influence the sex ratio and extinction risk of Taxus baccata? Plant Ecol 200: 229-240.

Kéry M, Matthies D, Schmid B (2003). Demographic stochasticity in population fragments of the declining distylous perennial Primula veris (Primulaceae). Basic Appl Ecol 4: 197-206. 
Klein EK, Lavigne C, Gouyon PH (2006). Mixing of propagules from discrete sources at long distance: comparing a dispersal tail to an exponential. BMC Ecol 6: 3 .

Koressaar T, Remm M (2007). Enhancements and modifications of primer design program Primer3. Bioinformatics 23: 1289-1291.

Leblois R, Rousset F, Estoup A (2004). Influence of spatial and temporal heterogeneities on the estimation of demographic parameters in a continuous population using individual microsatellite data. Genetics 166: 1081-1092.

Lee CL (1954). Sex chromosomes in Ginkgo biloba. Am J Bot 41: 545-549.

Litkowiec M, Plitta-Michalak BP, Lewandowski A, Iszkuło G (2015). Homogenous genetic structure in populations of Taxus baccata with varied proportions of male and female individuals. Silva Fenn 49 (no. 4)article id 1236.

Lyons EE, Waser NM, Price MV, Antonovics J, Motten AF (1989). Sources of variation in plant reproductive success and implications for concepts of sexual selection. Am Nat 134: 409-433.

Miao Y-C, Su J-R, Zhang Z-J, Li H, Luo J, Zhang Y-P (2008). Isolation and characterization of microsatellite markers for the endangered Taxus yunnanensis. Conserv Genet 9 : 1683-1685.

Mogensen HL (1996). The hows and whys of cytoplasmic inheritance in seed plants. Am J Bot 83: 383-404.

Nazareno AG, Alzate-Marin AL, Pereira RAS (2013). Dioecy, more than monoecy, affects plant spatial genetic structure: the case study of Ficus. Ecol Evol 3: 3495-3508.

Pannel RI, Bell PR (1988). Insemination of the archegonium and fertilization in Taxus baccata L. J Cell Sci 89: 551-559.
Petit RJ, Duminil J, Fineschi S, Hampe A, Salvini D, Vendramin GG (2005). Comparative organization of chloroplast, mitochondrial and nuclear diversity in plant populations. Mol Ecol 14: 689-701.

Rebould X, Zeyl C (1994). Organelle inheritance in plants. Heredity 72: 132-140.

Renner SS, Ricklefs RE (1995). Dioecy and its correlates in the flowering plants. Am J Bot 82: 596-606.

Rousset F (2000). Genetic differentiation between individuals. J Evol Biol 13. 58-62.

Saniga M (2000). Structure, production and regeneration processes of English yew in the State Nature Reserve Plavno. J For Sci 46: 76-90.

Schroeder JW, Tran HT, Dick CW (2014). Fine scale spatial genetic structure in Pouteria reticulata (Engl.) Eyma (Sapotaceae), a dioecious, vertebrate dispersed tropical rain forest tree species. Global Ecol Conserv 1: 43-49.

Suszka B (1985). Conditions for after-ripening and germination of seeds and for seedling emergence of English yew (Taxus baccata L.). Arb Kór 30: 285-338.

Vandepitte K, Honnay O, Meyer TD, Jacquemyn H, Roldán-Ruiz I (2010). Patterns of sex ratio variation and genetic diversity in the dioecious forest perennial Mercurialis perennis. Plant Ecol 206: 105-114.

Vekemans X, Hardy OJ (2004). New insights from fine-scale spatial genetic structure analyses in plant populations. $\mathrm{Mol} E \mathrm{ECO}$ 13: 921-935.

Vessela F, Salis A, Scire M, Piovesan G, Schirone B (2015). Natural regeneration and gender-specific spatial pattern of Taxus baccata in an old-growth population in Foresta Umbra (Italy). Dendrobiology 73: 75-90. 